\title{
Heart failure: Precision in diagnosis and therapy defining heart failure with preserved ejection fraction?
}

\author{
John D Rozich* \\ Tuomey Hospital, Sumter, SC, USA
}

\begin{abstract}
Clinicians use the term heart failure (HF) to identify patients suffering from inappropriate volume accumulation thought or found to be secondary to abnormalities in cardiac performance. Identifying its etiology enables delivery of suitable care in addition to prevention of recurrence. Failure to properly classify the syndrome of $\mathrm{HF}$ and its underlying cause may put vulnerable patients at risk. Recent efforts have delineated the important differences in etiologies, therapies and outcomes between $\mathrm{HF}$ with reduced left ventricular (LV) systolic performance, (HFrEF), versus HF with preserved LV ejection fraction (HFpEF). But consideration for even greater precision exists within the diagnosis of $\mathrm{HFpEF}$, if optimization of care is to be realized for this common and ever-growing disease process. This perspective has underscored the primary concern that the "heart" itself, in the presentation of HFpEF, may not play a direct causative role in either the etiology or the phenotypic expression of this form of "heart failure syndrome". The consequences of this imprecision are to enable potential misuse of valuable healthcare resources and to distort admission, epidemiologic and even reimbursement metrics within the preeminent focus of providing optimal care for this vulnerable population. The focus of the present work is to ask whether the orthodox use of the moniker "heart failure", a condition with both widely disparate causes and resulting therapies, remains functionally appropriate given that the blurring of etiologic specificity for HFpEF may then adversely impact outcomes.
\end{abstract}

\section{Introduction}

Clinicians address the syndrome of heart failure (HF) by identifying its etiology to enable initiation of appropriate therapy. This identification of cause is important in achieving delivery of optimal care and in preventing recurrence. Thus, failure to properly classify the syndrome of $\mathrm{HF}$ and its underlying cause may endanger vulnerable patients. Recent data has underscored the important differences in therapies and outcomes between HF with reduced left ventricular (LV) ejection fraction, (HFrEF), versus HF with preserved LV ejection fraction (HFpEF) [1]. Yet even with appreciation of these distinctions, there is growing recognition that further precision is required in understanding the contributing etiology and identifying appropriate treatments for patients suffering from the "heart failure syndrome", especially that wherein LVEF is preserved or labeled HFpEF.

This perspective has underscored the primary concern that the "heart" itself, in its presentation as HFpEF, may not play a direct causative role. Specifically, the etiology or the final expression of the "heart failure syndrome" as it applies to HFpEF may mislabel the myocardium as mechanistically determinative. Instead, the myocardial performance may be incidentally involved, or a co-participant lacking direct causation. This often creates a ripple effect, beginning with the potential for misuse of valuable healthcare resources. The ripple continues as summary admission, epidemiologic and even reimbursement metrics are distorted [2,3].

Experts in the field have long recognized the complexity involved in understanding the precise mechanism(s) responsible for HFpEF in each patient [4-6]. Further, disparate groupings of co-morbidities may be intimately involved in the more general phenotypic expression of HFpEF [5]. But in practice, clinicians routinely lump together patients with volume overload-related symptoms irrespective of their cause. The focus of the present work is to ask whether the orthodox use of the moniker "heart failure", a condition with both widely disparate causes and resulting therapies, remains appropriate given that the blurring of etiologic specificity may then adversely impact outcomes.

\section{The real world}

The American Heart Association defines heart failure as when "the heart isn't pumping as well as it should be".* The National Heart Lung and Blood institute notes that "heart failure is a condition in which the heart can't pump enough blood to meet the body's needs"** These are definitions that offer constructs that have guided clinicians in both diagnostic and therapeutic endeavors for several generations. Recognition that HF symptoms could be secondary to grossly preserved clinical myocardial performance, HFpEF, was not thoroughly understood initially, and it thus received comparatively little attention in comparison to classic systolic heart failure. But the last four decades have witnessed a robust examination of HFpEF's origins, therapies and outcomes suggesting that altered myocardial relaxation contributing to important hemodynamic pressure changes are involved [7]. In spite of these efforts confusion persists as to the actual role that abnormalities in specific diastolic cardiac performance play in HFpEF and its clinical outcomes [3].

\section{HFpEF Terminology in clinical practice}

The best contextual understanding of HFpEF is that it represents a syndrome with rather significant pathophysiological heterogeneity.

*Correspondence to: John D Rozich, Tuomey Hospital Sumter, SC 29150, USA, E-mail: johnrozich@hotmail.com

Key words: heart failure, left ventricular

Received: September 03, 2019; Accepted: September 24, 2019; Published: September 30, 2019 
It thus may be argued that identifying a single etiology or therapeutic approach for HFpEF may not be practical or even appropriate. But this is exactly the issue with continued use of this term as it has become known and utilized. HFpEF inherently "muddies the waters" and contextually attempts to fit multiple different etiologies within a "single size" when in fact the "heart" is frequently not the principal problem.

Beginning with the concern that HFpEF was historically believed to be caused almost exclusively by diastolic dysfunction of the heart, subsequent investigative efforts demonstrated that other factors contribute and may actually be dominant in its clinical presentation [8]. Thus, the central concern raised in the present effort is whether a more precise focus on the etiology of the syndrome of volume overload and pulmonary congestion, in the setting of preserved LVEF, actually benefits treatment or outcomes because such a practice may more readily identify responsible triggers of injury. Alternatively, when the heart is the principal contributing problem, the characterization of cause and outcome remains paramount.

Before any conclusion can be reached, the elements of a hypothesis calling for enhanced precision should be addressed. First, we ask if there are specific cardiac abnormalities causally responsible for HFpEF? $[1,4,5,8]$. Second if not mechanistically determinant, do cardiac abnormalities contribute to HFpEF? Third, if not directly contributing, is the syndrome of HFpEF actually a pathophysiologic syndrome where cardiac performance is impacted in response to non-cardiac targeted organ systems? This spans the spectrum of direct cardiac causality to that of innocent bystander. While some may view this as an unnecessary game of semantics, the disparities in current and especially in future treatment and prognosis suggest otherwise.

\section{Is HFpEF mechanistically dependent upon cardiac abnormalities?}

Thus, the first set of concerns to address is the entity of HFpEF as a valid mechanistically-driven cardiac-specific abnormality [7]. Counter arguments suggest that absent contributing coexisting morbidities, HFpEF is not all that common [10]. Does such a perspective have merit and is it consequential? In fact, it is well accepted that dyspnea may be related to volume overload, caused by renal failure, or by pulmonary disease or by perturbation of cardiac diastolic performance, either alone or in combination with any of the aforementioned conditions. Does HFpEF occur secondary to variables in isolation that are mechanistically dependent upon the myocardium? Or alternatively is the syndrome so tightly intertwined with contributing requisite coconditions, that assigning causality solely to the myocardium for the phenotypic expression of HFpEF unsupportable? And importantly, for clinicians, the ultimate purveyors of such labels, does any of this enhanced specificity in etiology or contributing variables matter in the care of their patients?

Different expert opinions suggest definitional confusion exists with the term HFpEF versus HF "normal" EF (HFNEF) but it is well established that the label Heart Failure fundamentally triggers recognition of its linkage to serious adverse events culminating in a potentially grave set of outcome metrics $[7,9,10]$. But an alternative perspective argues that labels of "critically ill" or "critically sick" support a similar justified level of concern but are also correspondingly devoid of pathological, etiological or therapeutic specificity. Thus, in looking for greater specificity are there specific cardiac abnormalities noted in HFpEF mechanistically responsible for its development?

\section{Cardiac changes in HFpEF}

Beginning with the frequent reliance on LVEF as the pivotal metric to define HF in diastolic or systolic clinical syndromes, its use has been shown to impart significant inherent limitations [11]. Preservation at rest of LVEF does not ensure a normal EF response during exercise or physiologic stress [12-14]. A reduction in systolic reserve has been shown to be unmasked when attempting to enhance or augment stroke volume in patients demonstrating variable HFpEF [15]. This directly impacts diastolic LV reserve since failed contractile potential imparts reduced annular motion and recoil to early diastolic performance [15]. But experts have also noted that EF itself as measured by echocardiography lacks sensitivity in systolic performance, even at rest compared to strain imaging quantifying myocardial deformation $[16,17]$.

Longitudinal strain specifically was abnormal in greater than half of studied patients and was associated with increased risk of CV death or HF hospitalization, is less load dependent than EF and more readily demonstrates early abnormalities in patients denoted to have HFpEF $[17,18]$. But this study, Shah et al., was limited by concern over the small subset of patients (compared to total enrolled TOPCAT population) being actually available for complete pre- and post-strain analysis [17]. In addition, the segregation of cohorts based on the limited strain data was then used to demonstrate statistically significant trends in LVEF quartiles from group I: $53.4+9.4$ through group IV: $64.4+5.5 \mathrm{p}<0.001$ mean LVEF $59.9+8.0(n=447)$ [17]. While appreciation of observed LVEF disparities between groups I and IV may be theoretically attractive, the actual degree of overlap existing even between these two groups, containing the greatest absolute differences in LVEF, is small. Arguably, realistically separating groups II and III and their respective observed LVEF values would be unlikely in the clinical realm. Thus, practically, it is unlikely that clinical reliance on metrics shunning LVEF will soon become commonplace, given its essential and accessible clinical role in rapidly segregating overall LV myocardial performance.

Other cardiac-specific variables have recently come to light that involve cellular and molecular perturbation. It is believed that comorbidities of obesity, HTN and metabolic syndrome engender inflammation that impact cellular transduction elements through altered NO production $[7,11,18]$. Correlative human LV biopsies show reduced cGMP and protein kinase G felt responsible for elevated forms of myocyte stiffness [19,20]. Calcium level abnormalities are also implicated with increased levels in the sarcomere revealing prolonged contraction and relaxation in comparison to non-HFpEF controls $[21,22]$. Titin, an important and perhaps dominant cellular dynamically regulated determinant of stiffness, is also elevated [21,23,24]. But direct human data is sparse, and criticism again emphasizes that several of these same changes (noted above) actually occur in cohorts of NORMAL aging hearts [20]. Thus, because there are no direct animal models of HFpEF, refining prioritization of pathologic versus "normal" for age-related perturbations casually linked to HFpEF, often remains speculative [20]. And finally, for such a common pathological entity confronting clinicians, this lack of a surrogate investigative tool for HFpEF is indeed as troubling as it is remarkable. It thus warrants repeating that the co-morbid conditions of obesity, HTN and metabolic syndrome linked to resultant inflammation may be the true determinative variables that impact cardiac diastolic performance. But beyond this general statement that combinations of co-morbidities heighten risk for HFpEF, how do we stratify what plays an essential role in HFpEF risk? Different approaches have been proposed to identify patients vulnerable to developing HFpEF. 
Use of one such tool is the H2FPEF score, used to assist in predicting HFpEF $[25,26]$ of interest is that only one out of the six variables employed exclusively involves cardiac diastology. This involves the filling pressure derived by Doppler echocardiography [26]. The other echo-derived variable, pulmonary artery systolic pressure (PASP), is related to diastology, but it is often not exclusively a diastolicdriven element since primary pulmonary pathology may contribute. Also noteworthy is the fact that all of these six H2FPEF criteria were correlations based on logistic regression and then validated against a separate test cohort [26]. It has not been tested or validated in a larger and more diverse population (it is a single center study) and this fact is appropriately noted as a potential limitation by the originating authors. Further, although hinted at, it is proper to address the potential that these two echocardiographic-derived elements, pulmonary artery systolic pressure (estimated to be greater than $35 \mathrm{mmHg}$ ), and E/e' ratios, are subject to inaccurate results if there is suboptimal image acquisition and interpretation. PASP may also temporally vary significantly, depending upon volume status. Underlining this concern is that fact that echo-derived metrics are among the most technician-dependent data acquired in medical imaging, and thus must be carefully screened as to their accuracy in the non-academic (real) world. Concerns regarding interobserver temporal variability for echo-derived metrics have been previously noted $[27,28]$. Simply stated, "Even good images do not guarantee accurate metrics".

Allowing for the concern of consistency or accuracy in echo-derived diastolic metrics, the expression of cardiac diastolic abnormalities as independent contributing variables to the development of HFpEF deserves comment. They are of uncertain relevance in each affected patient and arguably, may not be the driving or participating factors in the etiology of HF absent other active co-morbidities in a significant proportion (currently unknown) of patients [29,30]. Existing data support that the development of diastolic dysfunction is clearly a risk factor for developing HFpEF, but the two are not equivalent states $[7,22,30]$. Cardiac diastolic filling abnormalities are ubiquitous within normal age-related myocardial changes [31-33].

As a corollary, let us examine a hypothetical scenario: the act of a seventy year old female attempting to lift a 30 pound (lb) weight. The inability of a certain number of 70 year old individuals to lift $30 \mathrm{lbs}$ of weight may be associated with any number of abnormalities. These may include sarcopenia secondary to various etiologies, deconditioning, arthritis, altered muscle energetics or co-existing diseases that may contribute to vascular insufficiency. Both diabetes and renal disease, if present, may also contribute to substantial changes in muscle mass, tone and energetics. Each one of these conditions may reflect associated systemic abnormalities; all may contribute to or be associated with the inability to lift 30 pounds. But none is individually determinative or mechanistically responsible in each and every one of the many patients who will be unable to achieve the 30 pound weight lift. These changes are also present, with broad overlap, to those noted in normal aging.

Is not the syndrome of HFpEF similar? We are examining diastology, myocardial protein and energetic performance, fibrotic content along with inflammation and yes these are present to a greater extent in HFpEF than their absence. But they are also present in multiple patients without HFpEF and thus may actually hold little inherent specificity for the development of HFpEF [7,20,22,29]. Thus, diastolic performance of the heart within the context of HFpEF should not be dismissed as these metrics may contribute meaningful insights, but alone or even in combination with other variables, they are not per se causally responsible for HFpEF.

\section{Diastolic dysfunction versus $\mathrm{HFpEF}$}

It is important to re-emphasize that HFpEF does not equate to diastolic dysfunction either in the specific variables describing these two separate entities (HFpEF and diastolic dysfunction) or their respective clinical presentations $[11,13,20,21,29]$. There are numerous shared or common elements, but there is a definitive distinction between these two physiologic states. In essence diastolic dysfunction refers to mechanical properties of the ventricle in diastole that become altered (see above) whereas HFpEF denotes signs and symptoms of clinical HF in a patient with normal LVEF (most often determined by echocardiography), but one in which there is also LV diastolic dysfunction [21]. Through reduction in the heart rate an extension in the interval of diastole allows more effective sarcolemma calcium sequestration [11,21,22,34]. Elevated left atrial pressures causing elevated pulmonary pressures may stretch both atrial and ventricular tissue triggering a protective increased release of brain natriuretic peptide (BNP) and enhanced diuresis. Each of these steps provide safeguards in the form of compensatory adaptation to gradual altered diastology. When these steps cannot overcome destructive changes in cardiac adaptation to rate, rhythm or volume, the syndrome of HFpEF is manifest [22]. But the central question that this effort poses is what has caused the changes in rate, rhythm or volume in the first place?

\section{Contributing but not primarily responsible.}

And thus, it is fair to ask if the development of HFpEF is related to or dependent upon diastolic dysfunction? Or at times is it incidental? Using the following example, we ask is this HFpEF?

A 63 y.o. obese patient with T2DM and HTN presents with dyspnea, pulmonary congestion or early pulmonary edema related to acute intravascular volume overload. Diastolic filling abnormalities were detected on echocardiography, consisting of an elevated E/e' of 16; in accordance with established criteria the diagnosis is consistent with HFpEF. As it turned out this patient had acute renal decompensation, acute renal failure in the setting of diabetes and use of NSAIDs with no previous overt cardiac history and no evidence of changing cardiac performance. There is clinical volume overload, LVEF is $65 \%$ with borderline increased cardiac mass or LVH. Is there HFpEF?

Is this really HFpEF? And if diastolic abnormalities are detected, are these determinative in the development of the dyspnea for this patient? This is more than semantics or word play as the contribution of diastology or cardiac performance may actually be remarkably unimportant in the presentation of HFpEF when other dominant etiologies are in play. It is of interest however, that remarkably little exists in our investigative literature regarding the impact of inappropriate use of disease labels that may misinform or misdirect subsequent events [35].

\section{The role of inflammation and its progenitors}

Having addressed observed cardiac-specific abnormalities identified in HFpEF, it is reasonable to address the other associated elements that characterize the HFpEF state. The identification of obesity, diabetes, obstructive sleep apnea and hypertension noted previously often in the setting of contribution of tachydysrhythmias may be important variables resulting in or contributing to HFpEF $[7,22,24,30,31]$. But even to a casual observer, each of these contributing etiologies, either as single elements or in various combinations, also exist in many patients who never experience the HFpEF syndrome. Thus, there is an intense interest in what else could 
be occurring in vulnerable individuals that creates fertile ground for HFpEF.

Several lines of evidence suggest that while hypertension is known to trigger hypertrophic growth in the LV myocardium; alone it is not solely culpable in the development of HFpEF $[11,21,33]$. Other factors contribute and may indeed be essential in the ventricular remodeling and stiffening noted to play a role in HFpEF [11]. As identified earlier, disparate etiologies contribute to HFpEF and include the roles of obesity and insulin resistance. These are now proposed to produce or incite a proinflammatory setting that is thought to distort the more common and foreseeable type of hypertrophy (perhaps a more benign form?) resulting from hypertension alone [11]. The hypothesis that an activated inflammatory state may indeed alter phenotypic "behavior" of the heart resulting in changes to the interstitial tissue and individual myocytes themselves has been proposed [7,11,13,20-24]. The defining perturbations are at present incompletely defined, but converging lines of experimental data have identified multiple potential players. Excessive adiposity-generated bioactive agents may heighten inflammation that in turn induces fibrotic activity among subpopulations of reparative cells [36]. This coupled to downstream effects of insulin transduction may also directly negatively modify the ultrastructural integrity of cardiac myocytes, in addition to engendering direct fibrosis of the delicate existing infrastructure surrounding these cells in the myocardium $[(19,24,29,36,37]$. There are also separate lines of evidence emphasizing endothelial to mesenchymal transitional significance in response to nuanced molecular signaling that cause the pathologic phenotypic stiffness of observed abnormal myocardial diastology [38]. The result is increased stiffness, and a loss in the hemodynamic plasticity leading to more rapid increases in both chamber pressures and lung edema.

Obesity in particular appears to be a key risk factor and distinct phenotype of HFpEF. As compared with nonobese patients, obese HFpEF patients display greater degrees of volume overload, more right heart dysfunction and remodeling, increased levels of systemic inflammation, poorer exercise capacity, and greater ventricular interdependence with right heart pressure and volume changes influencing the left heart $[7,22]$. of these variables, it is the differential expression of levels of inflammation that again potentially underwrites a unifying concern. It may predispose patients to an intolerance to volume challenges that are not problematic for those with less sustained systemic inflammatory activity.

The expansion on this hypothesis relates that the proinflammatory state causes coronary microvascular endothelial cells to also produce reactive oxygen species that reduce nitric oxide bioavailability, which in turn decreases protein kinase $\mathrm{G}$ activity in cardiomyocytes $[39,40]$. Lowered protein kinase $G$ activity is proposed to disinhibit cardiomyocyte hypertrophy causing hypertrophic-associated inflexibility due to hypophosphorylation of the cytoskeletal protein titin [39]. Per this theoretical construct, coronary microvascular endothelial cells also produce vascular cell adhesion molecule and E-selectin, which promote migration of monocytes into the subendothelium. Transforming growth factor beta released by monocytes stimulates conversion of fibroblasts into myofibroblasts that deposit collagen in the interstitial space. The specific roles that each of these plays, or the degree of participation and combinations seen in HFpEF, remain to be illuminated. What appears to be the growing consensus, however, is that the inflammatory response, different in degree, etiology and substance in different individuals, may set the stage for a phenotypic transformation to HFpEF.

\section{Does specificity matter?}

But again, clinicians commonly face patients having all three of these risk factors, hypertension, obesity and diabetes, and to a varying degree associated inflammatory activation, but no evidence of HFpEF. None of these aforementioned conditions, (obesity, diabetes, obstructive sleep apnea and hypertension) originate in the heart, although each assuredly ultimately impacts cardiac function. The phenotypic presentation of an elderly patient in obvious respiratory distress demands urgent action and the prevailing approach is to reduce the intravascular volume and reestablish a comfortable baseline. To this point one may readily ask if specificity has any real value in this context?

Similar to high output failure, related to an arteriovenous (AV) fistula, reducing excessive volume is priority one, followed by investigation of cause; closing or treating the AV fistula. Cardiacdirected therapy here is discretionary or not relevant when considering the etiology. Every emergency department faces such challenges dozens of times a month. From a practical standpoint clinicians intervene in the setting of HFpEF and attempt to rapidly reverse the problem. It is the second phase of therapy, wherein use of several accessible variables to better define the source of HFpEF, to define etiology, that importance in specificity in cause begin to surface.

This question, as to the benefit of enhancing specificity in assigning an etiology and diagnosis of HFpEF, may itself require careful consideration. When in fact volume overload secondary to chronic kidney disease, or pulmonary disease causing peripheral edema and signs of volume excess are the actual culprits, is there a tangible benefit to more focused and correct identification of the actual etiology? At some point for each patient's optimal benefit the answer is of course, "it depends". Careful articulation of multiple abnormalities in each patient is likely to eventually identify the predominant cause of the volume excess. If the syndrome is one of HF and the LVEF is preserved, is there really a problem? It is the focus of this effort to propose that defining the causative etiology becomes a determinative variable in the future care of every patient. Lumping patients into a HFpEF without effort to identify source is potentially dangerous but unfortunately remains common . The consequences may become even more apparent as new lines of evidence are explored linked to new strategies to combat HFpEF [41].

\section{Conclusion}

It must be underscored that clarity in both seeking and understanding the etiology of HFpEF remains inconstant. Busy clinicians are treating patients with multiple sources of volume excess. In the era of hospitalists wherein episodic care may inadvertently create both "temporal and subspecialty silos", the energy allocated to clearly identifying the contributing comorbidities for intravascular volume overload may be incomplete. While detailed and extensive investigative efforts have begun to unmask the contributing variables found in $\mathrm{HFpEF}$, a preeminent observation has been that the syndrome of intravascular volume overload is multifaceted and often the myocardial contribution to such phenotypic volume excess is incidental; perhaps even minimal. It is time to first begin to address whether greater precision leads to enhanced outcomes. Only then can we debate whether the end of the era of labeling disparate sources of volume overload "heart failure" justified, as was the era wherein "dropsy" identified those with impaired LV systolic performance.

\section{References}

1. Tromp J, Westenbrink BD, Ouwerkerk W, van Veldhuisen DJ, Samani NJ, et al. (2018) Identifying pathophysiological mechanisms in heart failure with reduced versus preserved ejection fraction. J Am Coll Cardiol 72: 1081-1090. [Crossref] 
2. Fuat A1, Hungin AP, Murphy JJ (2003) Barriers to accurate diagnosis and effective management of heart failure in primary care: qualitative study. BMJ 326: 196. [Crossref]

3. Remes J, Miettinen H, Reunanen A, Pyorala K (1991) Validity of clinical diagnosis of heart failure in primary health care. Eur Heart $J$ 12: 315-321.

4. Zile MR (2003) Heart failure with preserved ejection fraction: is this diastolic heart failure? J Am Coll Cardiol 41: 1519-1522. [Crossref]

5. Vasan RS, Levy D (2000) Defining diastolic heart failure: a call for standardized diagnostic criteria. Circulation 101: 2118-2121.

6. Hunt SA, Baker DW, Chin MH (2001) ACC/AHA guidelines for the evaluation and management of chronic heart failure in the adult: Executive summary a report of the american college of cardiology/american heart association task force on practice guidelines (committee to revise the 1995 guidelines for the evaluation and management of heart failure): developed in collaboration with the international society for heart and lung transplantation; endorsed by the heart failure society of america. Circulation 104: 2996-3007.

7. Borlaug BA (2014) The pathophysiology of heart failure with preserved ejection fraction. Nat Rev Cardiol 11: 507-515. [Crossref]

8. Zile MR, Baicu CF, Gaasch WH (2004) Diastolic heart failure--abnormalities in active relaxation and passive stiffness of the left ventricle. N Engl J Med 350: 1953-1959. [Crossref]

9. Sanderson JE (2014) Reply: What is normal in HFNEF? The case for HFpEF. JACC Heart Fail 2: 543-544. [Crossref]

10. Sanderson JE (2014) HFNEF, HFpEF, HF-PEF, or DHF: what is in an acronym? JACC Heart Fail 2: 93-94. [Crossref]

11. Borlaug BA, Lam CS, Roger VL, Rodeheffer RJ, Redfield MM (2009) Contractility and ventricular systolic stiffening in hypertensive heart disease insights into the pathogenesis of heart failure with preserved ejection fraction. J Am Coll Cardiol 54: 410-418. [Crossref]

12. Shah AM, Claggett B, Folsom AR (2015) Ideal cardiovascular health during adult life and cardiovascular structure and function among the elderly. Circulation 132: 19791989.

13. Borlaug BA, Olson TP, Lam CS (2010) Global cardiovascular reserve dysfunction in heart failure with preserved ejection fraction. $J$ Am Coll Cardiol 56: 845-854.

14. Abudiab MM, Redfield MM, Melenovsky V (2013) Cardiac output response to exercise in relation to metabolic demand in heart failure with preserved ejection fraction. Eur $J$ Heart Fail 15: 776-785.

15. Borlaug BA, Kane GC, Melenovsky V, Olson TP (2016) Abnormal right ventricularpulmonary artery coupling with exercise in heart failure with preserved ejection fraction. Eur Heart J 37: 3293-3302.

16. Stokke TM, Hasselberg NE, Smedsrud MK (201) Geometry as a confounder when assessing ventricular systolic function: Comparison between ejection fraction and strain. J Am Coll Cardiol 70: 942-954.

17. Shah AM, Claggett B, Sweitzer NK (2015) Prognostic importance of impaired systolic function in heart failure with preserved ejection fraction and the impact of spironolactone. Circulation 132: 402-414.

18. Mentz RJ, Khouri MG (2015) Longitudinal strain in heart failure with preserved ejection fraction: is there a role for prognostication? Circulation 132: 368-370.

19. van Heerebeek L, Hamdani N, Falcão-Pires I, Leite-Moreira AF, Begieneman MP, et al. (2012) Low myocardial protein kinase G activity in heart failure with preserved ejection fraction. Circulation 126: 830-839. [Crossref]

20. Sharma K, Kass DA (2014) Heart failure with preserved ejection fraction: mechanisms, clinical features, and therapies. Circ Res 115: 79-96. [Crossref]
21. Borlaug BA, Paulus WJ (2011) Heart failure with preserved ejection fraction: pathophysiology, diagnosis, and treatment. Eur Heart J 32: 670-679.

22. Borlaug BA, Kass DA (2006) Mechanisms of diastolic dysfunction in heart failure Trends Cardiovasc Med 16: 273-279.

23. van Heerebeek L, Borbely A, Niessen HW (2006) Myocardial structure and function differ in systolic and diastolic heart failure. Circulation 113: 1966-1973.

24. Borbély A, van der Velden J, Papp Z, Bronzwaer JG, Edes I, et al. (2005) Cardiomyocyte stiffness in diastolic heart failure. Circulation 111: 774-781. [Crossref]

25. Paulus WJ (2018) H2FPEF Score. Circulation 138: 871-873. [Crossref]

26. Reddy YNV, Carter RE, Obokata M, Redfield MM, Borlaug BA (2018) A simple, evidence-based approach to help guide diagnosis of heart failure with preserved ejection fraction. Circulation 138: 861-870. [Crossref]

27. Otterstad JE (2002) Measuring left ventricular volume and ejection fraction with the biplane Simpson's method. Heart 88: 559-560. [Crossref]

28. Jenkins C, Chan J, Hanekom L, Marwick TH (2006) Accuracy and feasibility of online 3-dimensional echocardiography for measurement of left ventricular parameters. $J \mathrm{Am}$ Soc Echocardiogr 19: 1119-1128.

29. Shah SJ, Katz DH, Selvaraj S (2015) Phenomapping for novel classification of heart failure with preserved ejection fraction. Circulation 131: 269-279.

30. Borlaug BA (2016) Is HFpEF One Disease or Many? J Am Coll Cardiol 67: 671-673.

31. Arbab-Zadeh A, Dijk E, Prasad A, Fu Q, Torres P, et al. (2004) Effect of aging and physical activity on left ventricular compliance. Circulation 110: 1799-1805. [Crossref]

32. Fujimoto N, Hastings JL, Bhella PS, Shibata S, Gandhi NK, et al. (2012) Effect of ageing on left ventricular compliance and distensibility in healthy sedentary humans. $J$ Physiol 590: 1871-1880. [Crossref]

33. Borlaug BA, Redfield MM, Melenovsky V (2013) Longitudinal changes in left ventricular stiffness: a community-based study. Circ Heart Fail 6: 944-952.

34. Borlaug BA, Koepp KE, Melenovsky V (2016) Reply: Nitrites/Nitrates in heart failure with preserved ejection fraction. J Am Coll Cardiol 67: 1383-1384.

35. Trevethan R (2017) Sensitivity, specificity, and predictive values: foundations, pliabilities, and pitfalls in research and practice. Front Public Health 5: 307.

36. van Heerebeek L, Hamdani N, Handoko ML, Falcao-Pires I, Musters RJ, et al. (2008) Diastolic stiffness of the failing diabetic heart: importance of fibrosis, advanced glycation end products, and myocyte resting tension. Circulation 117: 43-51. [Crossref]

37. Shah AM, Claggett B, Sweitzer NK (2015) Prognostic importance of changes in cardiac structure and function in heart failure with preserved ejection fraction and the impact of spironolactone. Circ Heart Fail 8: 1052-1058.

38. Kovacic JC, Dimmeler S, Harvey RP, Finkel T, Aikawa E, et al. (2019) Endothelial to mesenchymal transition in cardiovascular disease: jacc state-of-the-art review. $J$ Am Coll Cardiol 73: 190-209. [Crossref]

39. Paulus WJ, Tschope C (2013) A novel paradigm for heart failure with preserved ejection fraction: comorbidities drive myocardial dysfunction and remodeling through coronary microvascular endothelial inflammation. J Am Coll Cardiol 62: 263-71.

40. Shah SJ, Kitzman DW, Borlaug BA, van Heerebeek L, Zile MR, et al. (2016) Phenotype-specific treatment of heart failure with preserved ejection fraction: a multiorgan roadmap. Circulation 134: 73-90. [Crossref]

41. Florijn BW, Bijkerk R, van der Veer EP (2018) Gender and cardiovascular disease: are sex-biased microRNA networks a driving force behind heart failure with preserved ejection fraction in women? Cardiovasc Res 114: 210-225. [Crossref]

Copyright: $\odot 2019$ Rozich JD. This is an open-access article distributed under the terms of the Creative Commons Attribution License, which permits unrestricted use, distribution, and reproduction in any medium, provided the original author and source are credited. 